Conclusions Transcriptomic analysis of Yaa mice before onset of SLE like clinical disease and DKO mice that develop accelerated disease uncovered differences in immune profiles, which point to a role for CD8 $\mathrm{T}$ cells in protection from autoimmunity in this model. In the absence of CD8 T cells, DKO mice exhibited an increase in gene signatures of M2-like M $\phi$ s representing a unique functional subset correlated with pathologic pathway signatures. This analysis provides evidence for a protective, regulatory function of CD8 T cells against autoimmune pathology with implications for understanding their role in human SLE.

\section{A STEPWISE TRANSCRIPTOMIC ANALYSIS USING GENE MODULES AND IMMUNE CELL SIGNATURES TO STRATIFY SYSTEMIC LUPUS ERYTHEMATOSUS PATIENTS AND IDENTIFY POTENTIAL TREATMENT TARGETS}

'Jozsef Karman*, 'Marc C Levesque, ${ }^{2} J u s t i n$ Wade Davis. 'AbbVie, Inc., Cambridge, MA, USA; ${ }^{2}$ AbbVie, Inc. North Chicago, IL, USA

\subsection{6/lupus-2021-lupus21century.12}

Background A major challenge in drug development for systemic lupus erythematosus (SLE) is the heterogeneous clinical presentation of SLE patients, which necessitates personalized treatment strategies. We aimed to identify clusters of SLE patients based on molecular transcriptomic signatures associated with clinical phenotypes to help address this challenge.

Methods To address this question, we developed an integrated pipeline that defines subsets of patients based on cell type-specific gene expression in blood. Gene expression profiles from two large independent SLE trials, ILLUMINATE-1 and ILLUMINATE-2, were analyzed to identify SLE patient clusters. We first performed a gene expression correlation network analysis to identify co-expressed gene modules. Then, unsupervised consensus clustering was performed on the modules to identify molecular clusters. We correlated cluster membership with clinical phenotypes and immune cell signatures from high resolution scRNA-seq data. We also determined whether immune cell signatures were stable over time.

Results We identified four molecular clusters of SLE patients. Cluster 1 exhibited high signature scores for T cells, B cells, plasma cells, macrophages, and monocytes. Conversely, Cluster 2 exhibited low signature scores for the aforementioned cells.

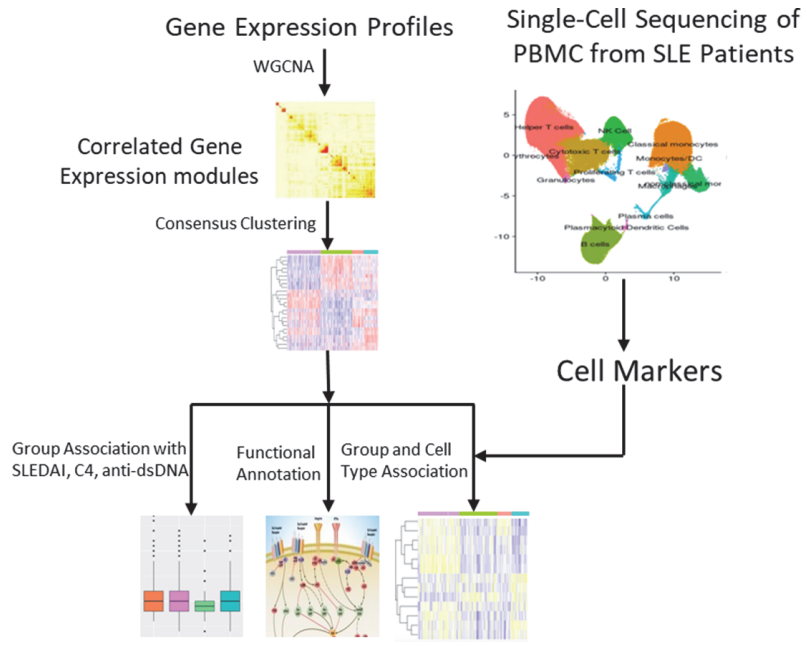

Abstract 303 Figure 1 Overview of analysis
Cluster 3 had high $\mathrm{T}$ and $\mathrm{B}$ cell signature scores. Cluster 4 had a high signature score for neutrophils. Clinically, Cluster 3 subjects exhibited the lowest disease severity compared to other clusters. We validated these four molecular clusters in three additional independent SLE cohorts. We identified four molecular clusters of SLE patients that were consistent across five independent genomics datasets totaling 2,100 patients. For individual patients, cluster membership was not necessarily stable over time.

Conclusions We have established methods to address SLE heterogeneity in a data-driven, unbiased manner using transcriptomic data. We have uncovered reproducible patterns in stratifying SLE patients using this method and connected SLE patient subsets to cellular alterations in the blood. Our findings have important implications for personalized treatment of SLE and provide guidance for clinical trials in this highly heterogeneous disease.

Acknowledgments Yingtao $\mathrm{Bi}$, Eric Yang, Jesus Paez-Cortez, Abel Suarez-Fueyo, Rui Wang (AbbVie; for suggestions on data analysis and review of results); Yingchun Liu, Stephen Clarke, Sherry Cao (Former AbbVie; for suggestions on data analysis and review of results).

\section{METABOLIC DYSREGULATION CHARACTERIZES THE TISSUE RESPONSE TO IMMUNE INJURY IN SYSTEMIC LUPUS ERYTHEMATOSUS AND INFLAMMATORY SKIN DISEASES}

${ }^{1}$ Kathryn M Kingsmore*, ' ${ }^{1}$ neha Shrotri, ${ }^{1}$ Brittany A Martinez, ${ }^{1}$ Prathyusha Bachali, ${ }^{1,2}$ Michelle D Catalina, ${ }^{1}$ Andrea R Daamen, ${ }^{1,3}$ Sarah E Heuer, ${ }^{1}$ Robert D Robl, ${ }^{1}$ Amrie C Grammer, ${ }^{1}$ Peter E Lipsky. ${ }^{1}$ AMPEL BioSolutions, LLC and RILITE Research Institute, Charlottesville, VA, USA; ${ }^{2}$ EMD Serono Research and Development Institute, $45 \mathrm{~A}$ Middlesex Turnpike, Billerica, MA 01821, USA; ${ }^{3}$ The Jackson Laboratory, Tufts Graduate School of Biomedical Sciences, 600 Main Street Bar, Harbor, ME 04609, USA

\subsection{6/lupus-2021-lupus21century. 13}

Background Autoimmune and inflammatory diseases such as systemic lupus erythematous (SLE) affect tissues throughout the body. Alterations to inflammatory cell metabolism are often cited as a contributing factor in diseases such as lupus ${ }^{1}$, however, changes to metabolism in diseased tissues are poorly understood. Therefore, we investigated changes to cellular metabolic processes in the tissues affected by SLE as well as samples from other inflammatory skin diseases.

Methods Gene expression data collected from patients with lupus nephritis (LN) glomerulus (GL), LN tubulointerstitium (TI), discoid lupus erythematosus (DLE), psoriasis (PSO), atopic dermatitis (AD), and systemic sclerosis (SSc), or murine LN was obtained from Gene Expression Omnibus. Enrichment of metabolic and cellular signature in individual samples was analyzed using Gene Set Variation Analysis (GSVA). Stepwise regression and classification and regression tree (CART) analyses were performed to determine correlations between each metabolic signature and all cellular signatures in each diseased tissue.

Results Comprehensive gene expression analysis of samples derived from glomerular and tubulointerstitial LN kidneys, and DLE, PSO, AD, and SSc skin revealed concurrent changes to genes reflective of cellular metabolic processes and cellular transcripts. In lupus-affected tissues there were shared decreases to metabolic gene signatures ${ }^{2}$, whereas in other inflammatory skin diseases some metabolic transcripts were increased. In glomerular LN, decreased glycolysis gene expression was correlated with increased endothelial cell transcripts ${ }^{2}$. 